\title{
COMBINATORIAL INEQUALITIES AND SMOOTHNESS OF FUNCTIONS
}

BY ADRIANO M. GARSIA ${ }^{1}$

Introduction. This is an expository account of work we have carried out jointly with some of our students and associates, notably E. Rodemich, $\mathrm{H}$. Taylor, C. Preston, C. Greenhall, S. Milne, T. Park and P. DeLand.

We shall be concerned with classes of functions $f(X)$ measurable on the $d$-dimensional unit cube

$$
I_{d}=[0,1] \times[0,1] \times \cdots \times[0,1]
$$

and satisfying conditions of the type

$$
I_{\Psi, p}(f)=\int_{I_{d}} \int_{I_{d}} \Psi\left(\frac{f(X)-f(Y)}{p(\overline{X Y})}\right) d X d Y \leqq B<\infty, 2
$$

where $\Psi$ and $p$ are restricted as follows:

and

(a) $\Psi(u)$ is defined and continuous on $(-\infty,+\infty)$,

(b) $\Psi(u)=\Psi(-u) \uparrow \infty$ as $|u| \uparrow \infty$

(a) $p(u)$ is defined and continuous on $(-\sqrt{ } d, \sqrt{ } d)$,

(b) $p(u)=p(-u) \downarrow 0$ as $|u| \downarrow 0$.

In most of our work we have been concerned with the one-dimensional case, but here we shall be able to say something about the general case.

Roughly speaking, our aim has been to use the finiteness of $I_{\Psi, p}(f)$ to derive a priori bounds for other important functionals of $f$, such as its modulus of continuity or other high order norms. The bounds we have obtained involve, of course, $\Psi$ and $p$ but they depend on $f$ only through the value of $I_{\Psi, p}(f)$.

1. Sample of results. To get across at least the flavor of our work we shall give a sample of our results in the one-dimensional case.

THEOREM 1.1. Let

$$
I_{\Psi, p}(f)=\int_{0}^{1} \int_{0}^{1} \Psi\left(\frac{f(x)-f(y)}{p(x-y)}\right) d x d y \leqq B<\infty,
$$

An address delivered at the 80th Annual Meeting of the American Mathematical Society in San Francisco on January 15, 1974; received January 20, 1975.

AMS (MOS) subject classifications (1970). Primary 26A54, 26A15, 42 A16.

${ }^{1}$ Work supported in part by Air Force Grant AF AFOSR 1322-67 and in part by NSF Grant.

\footnotetext{
${ }^{2} \overline{X Y}$ denotes the Euclidean distance from $X$ to $Y$.
} 
and suppose

$$
\int_{0}^{1} \Psi^{-1}\left(B / u^{2}\right) d p(u)<\infty ;^{3}
$$

then $f$ is essentially continuous and, in fact, for all $x$ and $y$ in the Lebesgue set of $f$ we have

$$
|f(x)-f(y)| \leqq 8 \int_{0}^{|x-y|} \Psi^{-1}\left(B / u^{2}\right) d p(u) .
$$

Several interesting applications of this theorem have been found since its discovery. (See [6], [7] and [1].) Indeed, our initial motivation for proving such a result came from a study of path continuity of Gaussian processes where the particular case $\Psi(u)=\exp u^{2}$ turns out to be very useful.

Theorem 1.1 has been the first result and our further work was stimulated by the puzzling question of what we could say about $f$ when $I_{\Psi, p}(f)<\infty$ and $\int_{0}^{1} \Psi^{-1}\left(B / u^{2}\right) d p(u)=\infty \quad \forall B$. It can be easily shown by examples that the $f^{\prime} s$ satisfying (1.1) need not be continuous in this case. Nevertheless, it is reasonable to ask how much smoothness is still present.

During the last few years we have put together a certain number of results in this direction (see [1], [5] and [2]). As an example in point we state an inequality which is true in general and implies (1.2) when condition (1.1) is satisfied.

To this end we recall that to each $f$ we can associate what is commonly called its "monotone rearrangement", denoted by $f^{*}$, which is defined as the unique nonincreasing function on $[0,1]$ such that

$$
m\{x: f(x) \geqq \lambda\}=m\left\{x: f^{*}(x) \geqq \lambda\right\} \quad \forall \text { real } \lambda .
$$

The basic inequality can be stated as follows:

THEOREM 1.2. If $I_{\Psi, p}(f) \leqq B<\infty$ and $\Psi\left(e^{x}\right)$ is convex, then for $0<x \leqq \frac{1}{2}$,

$$
\left.\begin{array}{c}
f^{*}(x)-f^{*}\left(\frac{1}{2}\right) \\
f^{*}\left(\frac{1}{2}\right)-f^{*}(1-x)
\end{array}\right\} \leqq 4 \int_{x}^{1 / 2} \Psi^{-1}\left(\frac{B}{u^{2}}\right) d p(u)+4 \Psi^{-1}\left(\frac{B}{x^{2}}\right) p(x) .
$$

A certain number of interesting inequalities can be derived from (1.3). In addition, Theorem 1.2 implies Theorem 1.1 at least when $\Psi\left(e^{x}\right)$ is convex.

However, the crucial fact which is responsible for (1.3) as well as several remarkable inequalities is that the functionals $I_{\Psi, p}(f)$ decrease when we replace $f$ by $f^{*}$. Namely we always have

$$
I_{\Psi, p}\left(f^{*}\right) \leqq I_{\Psi, p}(f) .
$$

Thus we can systematically reduce the study of $f$ to that of $f^{*}$ and the latter is simpler since $f^{*}$ is monotone.

We cannot escape the temptation to state here at least two of the most curious consequences of (1.4) which do not follow from (1.3).

\footnotetext{
${ }^{3} \Psi^{-1}$ denotes the inverse function of $\Psi$ in $(0, \infty)$.
} 
THEOREM 1.3. Let

then

$$
\int_{0}^{1} \int_{0}^{1} \frac{|f(x)-f(y)|^{\alpha}}{|x-y|^{2}} d x d y \leqq B^{\alpha}<\infty \quad(\alpha>1)
$$

where

$$
\int_{0}^{1} \exp \left\{c_{\alpha} \frac{\left|f(x)-f^{*}\left(\frac{1}{2}\right)\right|^{\beta}}{B}\right\} d x d y \leqq 2 \sqrt{ } 2,
$$

THEOREM 1.4. If

$$
c_{\alpha}=(\log 2 / 2)^{\beta}, \quad \beta=\alpha /(\alpha-1) .
$$

$$
\int_{0}^{1} \int_{0}^{1} \exp \left\{\frac{|f(x)-f(y)|}{B} \log \frac{1}{|x-y|}\right\} d x d y<\infty,
$$

then $f$ is essentially Lipschitzian and, indeed,

$$
|f(x)-f(y)| \leqq B|x-y|
$$

must hold for all $x, y$ in the Lebesgue set of $f$.

REMARK. So far the only proof we know of (1.5) is via the inequality in (1.4). We are offering a bottle of Scotch for the first proof of (1.5) which does not use (1.4). ${ }^{4}$

The basic inequality which implies the monotonicity of our functionals $I_{\Psi, p}(f)$ in the one-dimensional case can be stated as follows:

LEMMA 1.1. Let $\Phi(u)=\Phi(-u) \uparrow$ as $|u| \uparrow$; then for $0<\delta<1$ we have

$$
\iint_{|x-y| \leqq \delta} \Phi\left(f^{*}(x)-f^{*}(y)\right) d x d y \leqq \iint_{|x-y| \leqq \delta} \Phi(f(x)-f(y)) d x d y .
$$

Quite recently we have discovered some very interesting consequences of this inequality. But we shall postpone stating these until the end. Let us just say here that the $d$-dimensional version of (1.6) implies some rather remarkable new and classical Sobolev type inequalities. These inequalities have something to do with the fact that (1.6) enables us to estimate the growth of $f^{*}(x)$ as $x \rightarrow 0^{+}$or $x \rightarrow 1^{-}$in terms of the behavior as $\delta \rightarrow 0$ of a given integral modulus of continuity of $f$ (such as, for instance, the $L_{p}$ modulus of continuity $\left.\omega_{p}(\delta, f)\right)$. But it is more exciting at this point to get first an idea of the type of combinatorial results that are responsible for (1.6).

2. The combinatorial inequalities. To show (1.6) we need only establish a discretized version, namely

THEOREM 2.1. Let $\Phi(u)=\Phi(-u) \uparrow$ as $|u| \uparrow$. Then for all real numbers $\lambda_{1}<\lambda_{2}<\cdots<\lambda_{n}$ and for all $1 \leqq M \leqq n$ we have

$$
\sum_{|i-j| \leqq M} \Phi\left(\lambda_{i}-\lambda_{j}\right) \leqq \sum_{|i-j| \leqq M} \Phi(f(i)-f(j))
$$

where $f$ is any function on $(1,2, \cdots, n)$ which takes the values $\lambda_{1}, \lambda_{2}, \cdots, \lambda_{n}$.

\footnotetext{
${ }^{4}$ The bottle of Scotch was won by L. Nirenberg who solved this problem a day after the talk.
} 
To understand the significance of this inequality let us rewrite it in a form which can be easily extended to the higher-dimensional case as well as to more general situations.

Let $(\Omega, \delta)$ be a discrete metric space. For instance:

(1) In the one-dimensional case we want to take

$$
\Omega=(1,2, \cdots, n), \quad \delta(i, j)=|i-j| .
$$

(2) In the two-dimensional case

$$
\Omega=(1,2, \cdots, n) \times(1,2, \cdots, n), \quad \delta(P, Q)=\overline{P Q} \quad \text { (Euclidean) }
$$

etc.

Let $N=|\Omega|=$ cardinality of $\Omega$. This given, we can define a function $f$ on $\Omega$ starting from its values $\lambda_{1}<\lambda_{2}<\cdots<\lambda_{N}$ (we can assume without loss that they are all different) by saying where in $\Omega$ we put the smallest (i.e. $\lambda_{1}$ ), where we put the next smallest (i.e. $\lambda_{2}$ ) etc.

In other words, each function on $\Omega$ can be considered made up of its values $\lambda_{1}, \lambda_{2}, \cdots, \lambda_{N}$ together with an assignment or rule for putting these numbers on $\Omega$. Such an assignment can be given simply as a map $\pi:(1,2, \cdots, N) \leftrightarrow \Omega$.

Keeping this in mind, for a given function $f(P)$ defined on $\Omega$, the expression

$$
\sum_{\delta(P, Q) \leqq M ; P, Q \in \Omega} \Phi(f(P)-f(Q))
$$

can be written in the form

$$
\sum_{\delta(\pi(i), \pi(j)) \leqq M} \Phi\left(\lambda_{i}-\lambda_{j}\right)
$$

where this sum is carried out over all couples $(i, j) \in$ $[1,2, \cdots, N] \times[1,2, \cdots, N]$ such that $\delta(\pi(i), \pi(j)) \leqq M$.

We can better visualize the situation if we introduce a directed graph which we shall call $G(N)$ and which is defined as follows. Its vertices are the couples $a=(i, j), 1 \leqq i<j \leqq N$, and between $a=(i, j)$ and $a^{\prime}=\left(i^{\prime}, j^{\prime}\right)$ we put an arrow going from $a$ to $a^{\prime}$ if and only if

$$
i^{\prime}=i-1, \quad j^{\prime}=j
$$

or

$$
i^{\prime}=i, \quad j^{\prime}=j+1 .
$$

In the figure below we have illustrated $G(6)$.

Furthermore we make the vertices of $G(N)$ into a partially ordered set by setting

$$
a=(i, j)<a^{\prime}=\left(i^{\prime}, j^{\prime}\right)
$$

if and only if $i^{\prime} \leqq i<j \leqq j^{\prime}$. 


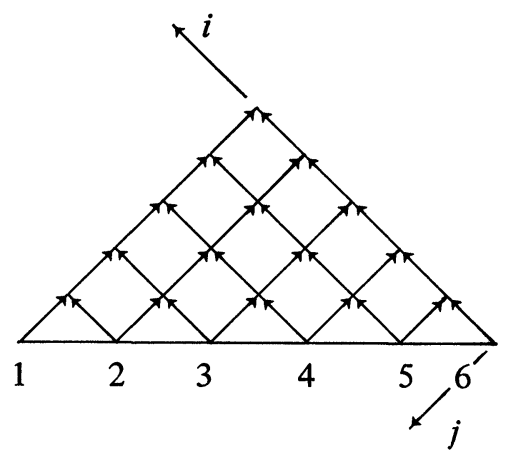

That is $a<a^{\prime}$ if and only if we can go from $a$ to $a^{\prime}$ in $G(N)$ along a path which follows the arrows.

Now, given the $\lambda$ 's and $\Phi$ as in Theorem 2.1 let us set for $a=(i, j)$, $\varphi(a)=\Phi\left(\lambda_{i}-\lambda_{j}\right)$. Note that this function is nondecreasing with respect to the partial ordering we have just introduced. This given, we rewrite $(2.3)$ in the form

$$
\sum_{a \in \mathscr{D}_{M}(\pi)} \varphi(a)
$$

where we have set

$$
\mathscr{D}_{M}(\pi)=\{a=(i, j): \delta(\pi(i), \pi(j)) \leqq M\} .
$$

The question then is: What is the smallest possible value that the expression in (2.5) can take when $\varphi(a)=\Phi\left(\lambda_{i}-\lambda_{j}\right)$ and $\pi$ varies amongst all possible ways of distributing $\lambda_{1}, \lambda_{2}, \cdots, \lambda_{N}$ in $\Omega$ ?

In the one-dimensional case, i.e. when $\Omega=(1,2, \cdots, n)$, there are two natural assignments, namely, $\pi(i)=i$ or $\pi(i)=n-i$. Indeed, Theorem 2.1 simply says that the smallest possible value of $(2.5)$ is obtained for any one of these two choices of $\pi$.

The proof of this fact, although at first sight it may seem to have to involve $\Phi$ and $\lambda_{1}, \lambda_{2}, \cdots, \lambda_{n}$, can actually be obtained by purely combinatorial considerations.

To see this, let us introduce one further notation. For two given subsets $A$ and $B$ of $G(N)$ we set $A<B$ if and only if $A$ is "below" $B$ in $G(N)$. By this we mean that there exists a one-to-one map $\theta$ of $A$ into $B$ which is increasing with respect to the partial order we introduced in (2.4). This given, we have the following crucial fact:

LEMMA 2.1. If $A$ and $B$ are two subsets of vertices of $G(N)$ then $\sum_{a \in \mathrm{A}} \varphi(a) \leqq \sum_{a \in \mathrm{B}} \varphi(a)$ holds for all $\varphi(a)=\Phi\left(\lambda_{i}-\lambda_{j}\right)$ if and only if $A<B$.

This is not difficult to prove if we use the P. Hall theorem on the selection of distinct representatives (the "marriage" theorem). Indeed, all we need to show is that our functions $\varphi(a)$ contain all the extreme points of the cone of increasing functions on $G(N)$. A proof of Lemma 2.1 along these lines is given in [5]. 
Set then for convenience

$$
\mathscr{R}_{M}=\{a=(i, j): 1 \leqq j-i \leqq M\} .
$$

If $\varepsilon$ denotes the identity mapping from $[1,2, \cdots, n]$ to $[1,2, \cdots, n]$ we see that

$$
\mathscr{R}_{M}=\mathscr{D}_{M}(\varepsilon) \text {. }
$$

From Lemma 2.1 we get that our inequality (2.1) is equivalent to the combinatorial inequality

$$
\mathscr{D}_{M}(\varepsilon)<\mathscr{D}_{M}(\pi) \quad \forall \pi
$$

or, which is the same,

$$
\mathscr{R}_{M}<\mathscr{D}_{M}(\pi) \quad \forall \pi .
$$

A proof of (2.1) via (2.6) can be found in [5]. In that paper quite a number of analytic inequalities are derived from (2.6) including Theorems 1.2, 1.3 and 1.4. But let us see next how all these results can be extended to the higher dimensional case.

For instance, let $\Omega=[1,2, \cdots, n] \times[1,2, \cdots, n]$ and

$$
\delta\left(P_{1}, P_{2}\right)=\overline{P_{1} P_{2}} \quad \text { (Euclidean). }
$$

That is, if $P_{1}=\left(i_{1}, j_{1}\right), P_{2}=\left(i_{2}, j_{2}\right)$ then

$$
\delta\left(P_{1}, P_{2}\right)=\sqrt{\left(i_{1}-i_{2}\right)^{2}+\left(j_{1}-j_{2}\right)^{2}} .
$$

In this case $N=|\Omega|=n^{2}$, so we want to represent our sum

$$
S=\sum_{P_{1} P_{2} \leqq M} \Phi\left(f\left(P_{1}\right)-f\left(P_{2}\right)\right)
$$

as a sum over a subset of the vertices of $G\left(n^{2}\right)$. With our notation, letting $\lambda_{1}<\lambda_{2}<\cdots<\lambda_{n^{2}}$ be the values taken by $f(P)$ in $\Omega$, and assuming that $f(P)$ takes the value $\lambda_{i}$ at the point $\pi(i)$ of $\Omega$, then

where

$$
S=\sum_{a \in \mathscr{D}_{\mathrm{M}}(\pi)} \varphi(a)
$$

$$
\mathscr{D}_{M}(\pi)=\{a=(i, j): \overline{\pi(i) \pi(j)} \leqq M\} .
$$

If we proceed in the most naive way, using the one-dimensional case as a guide, we might be tempted to ask whether or not there is a special map

$$
\varepsilon:\left[1,2, \cdots, n^{2}\right] \leftrightarrow \Omega=[1,2, \cdots, n] \times[1,2, \cdots, n]
$$

such that

$$
\sum_{a \in \mathscr{D}_{M}(\varepsilon)} \varphi(a) \leqq \sum_{a \in \mathscr{D}_{M}(\pi)} \varphi(a) \quad \forall \pi, \varphi \& M
$$

In view of Lemma 2.1 this is equivalent to

$$
\mathscr{D}_{M}(\varepsilon)<\mathscr{D}_{M}(\pi) \quad \forall \pi \& M \text {. }
$$


Going back to our sums, this would mean that given any function $f(P)$ on $\Omega=[1,2, \cdots, n] \times[1,2, \cdots, n]$ there exists a special rearrangement $f^{*}(P)$ of $f(P)$ such that

$$
\sum_{P_{1} P_{2} \leqq M} \Phi\left(f^{*}\left(P_{1}\right)-f^{*}\left(P_{2}\right)\right) \leqq \sum_{\frac{P_{1} P_{2} \leqq M}{}} \Phi\left(f\left(P_{1}\right)-f\left(P_{2}\right)\right) \quad \forall M .
$$

This would imply that for each function $f(x, y)$ on the square $I_{2}=[0,1] \times[0,1]$ there is a rearrangement $f^{*}(x, y)$ such that

$$
\begin{aligned}
& \iiint_{\sqrt{\left(x_{1}-x_{2}\right)^{2}+\left(y_{1}-y_{2}\right)^{2}} \leqq \delta} \Phi\left(f^{*}\left(x_{1}, y_{1}\right)-f^{*}\left(x_{2}, y_{2}\right)\right) d x_{1} d y_{1} d x_{2} d y_{2} \\
& \leqq \\
& \iiint_{\sqrt{\left(x_{1}-x_{2}\right)^{2}+\left(y_{1}-y_{2}\right)^{2}} \leqq \delta} \Phi\left(f\left(x_{1}, y_{1}\right)-f\left(x_{2}, y_{2}\right)\right) d x_{1} d y_{1} d x_{2} d y_{2} \quad \forall 0<\delta<\sqrt{ } 2 .
\end{aligned}
$$

Now, it requires little familiarity with the realities of higher dimensions to suspect that no such privileged rearrangements do exist, and to conclude that our search for the special map $\varepsilon$ in the inequality (2.8) is doomed to failure.

There is however, one further natural approach to extending our inequality (1.6) to the higher-dimensional case. That is, if we drop trying to rearrange $f(x, y)$ within the square, we might still try to see if an inequality similar to (2.9) may hold between $f(x, y)$ and its one-dimensional rearrangement. This rearrangement is defined as the nonincreasing function $f^{*}(s)$ in $[0,1]$ such that

$$
m\left\{s \in[0,1]: f^{*}(s) \geqq \lambda\right\}=m\{(x, y) \in[0,1] \times[0,1]: f(x, y) \geqq \lambda\} .
$$

But here we run into yet another problem. Namely, what to put for "??" in

$$
\begin{aligned}
\iint_{|s-t| \leqq ?} \Phi\left(f^{*}(s)-f^{*}(t)\right) d s d t & \\
& \leqq \iint_{\sqrt{\left(x_{1}-x_{2}\right)^{2}+\left(y_{1}-y_{2}\right)^{2}} \leqq \delta} \Phi\left(f\left(x_{1}, y_{1}\right)-f\left(x_{2}, y_{2}\right)\right) d x_{1} d y_{1} d x_{2} d y_{2} .
\end{aligned}
$$

From this viewpoint this approach appears even more hopeless, since it is hard to see how there can be any relation between distances in the square $[0,1] \times[0,1]$ and distances in $[0,1]$ that we could use to make sense out of (2.11).

Surprisingly enough, both approaches can be pushed through, and believe it or not they both lead to the same final inequality.

To see how this comes about let us go back to our inequality (2.8). Indeed, better yet, let us see what conditions a subset $A$ of vertices of $G\left(n^{2}\right)$ of the proper cardinality has to satisfy so that we may have

$$
A<\mathscr{D}_{M}(\pi)
$$

for all one-to-one maps $\pi$ of $\left[1,2, \cdots, n^{2}\right]$ into the two-dimensional lattice

$$
\mathscr{L}_{2}(n)=[1,2, \cdots, n] \times[1,2, \cdots, n] .
$$


To this end note that given any $0 \leqq i_{0} \leqq n^{2}-M^{2} / 2$ we can find a map $\pi_{0}:\left[1,2, \cdots, n^{2}\right] \leftrightarrow \mathscr{L}_{2}(n)$ which sends all the points $i_{0}+1, i_{0}+2, \cdots, i_{0}+M^{2} / 2$ into a fixed subsquare of $\mathscr{L}_{2}(n)$ whose diagonal is equal to $M$.

Clearly for such a map $\pi_{0}$, the set $\mathscr{D}_{M}\left(\pi_{0}\right)$ will necessarily contain the triangle $\mathscr{C}$ of vertices of $G\left(n^{2}\right)$ given by

$$
\mathscr{C}=\left\{a=(i, j): i_{0}+1 \leqq i<j \leqq i_{0}+M^{2} / 2\right\} .
$$

We claim that if (2.12) is to hold for $\pi=\pi_{0}$, in the sense that there is a point of $A$ below every point of $\mathscr{D}_{M}\left(\pi_{0}\right)$, then $A$ must also contain $\mathscr{C}$.

The reason for this is that there is no subset of vertices of $G\left(n^{2}\right)$ which has the same cardinality as $\mathscr{C}$ and lies below $\mathscr{C}$ other than $\mathscr{C}$ itself.

This reasoning carried out for every $i_{0}$ implies that if there is a map $\varepsilon:\left[1,2, \cdots, n^{2}\right] \leftrightarrow \mathscr{L}_{2}(n)$ such that $(2.8)$ holds $\forall \pi$, then $\mathscr{D}_{M}(\varepsilon)$ must contain the set $\mathscr{R}_{M^{2} / 2}$, and therefore we must also have

$$
\mathscr{R}_{\mathrm{M}^{2} / 2}<\mathscr{D}_{\mathrm{M}}(\pi) \quad \forall \pi \text {. }
$$

Conversely, suppose that for some constant $c$ (independent of $n$ ) we have

$$
\mathscr{R}_{M^{2} / \mathrm{c}}<\mathscr{D}_{\mathrm{M}}(\pi) \quad \forall \pi \& M \text {. }
$$

Then from Lemma 2.1 we get

$$
\sum_{|i-j| \leqq M^{2} / c} \Phi\left(\lambda_{i}-\lambda_{j}\right) \leqq \sum_{\overline{P Q} \leqq M} \Phi(f(P)-f(Q)) \quad \forall M
$$

for every function $f(P)$ on $\mathscr{L}_{2}(n)$ taking the values $\lambda_{1}, \lambda_{2}, \cdots, \lambda_{n^{2}}$.

It is not difficult to deduce, by passing to the limit, that the continuous version of the discrete inequality in (2.14) is

$$
\iint \Phi\left(f^{*}(s)-f^{*}(t)\right) d s d t \leqq \iint_{P Q} \Phi \delta(f(P)-f(Q)) d P d Q \quad \forall 0 \leqq \delta \leqq \sqrt{ } 2
$$

where $f^{*}(s)$ is the one-dimensional rearrangement of $f(P)$. So we see that this reasoning leads us to the value $\delta^{2} / c$ for "?" in (2.11).

This should shed some light upon the riddle concerning "the relation between distances in $[0,1] \times[0,1]$ and distances in $[0,1]$ "' we were wondering about. The relation between $\delta$ and "?" has a purely combinatorial origin. As we might easily guess at this point the $d$-dimensional analogue of $(2.15)$ is

$$
\text { 6) } \iint_{|s-t| \leqq \delta^{d} / c_{d}} \Phi\left(f^{*}(s)-f^{*}(t)\right) d s d t \leqq \iint_{P Q} \int_{\delta \delta} \Phi(f(P)-f(Q)) d P d Q, \quad 0 \leqq \delta \leqq \sqrt{ } d \text {, }
$$

and this, just like (2.15), can be derived from the combinatorial inequality

$$
\mathscr{R}_{M^{d} / c_{d}}<\mathscr{D}_{M}(\pi) \quad \forall \pi, M
$$

where, of course, now $\mathscr{R}_{M^{d} / c_{d}}$ and $\mathscr{D}_{M}(\pi)$ denote subsets of vertices of $G\left(n^{d}\right)$ and $\pi$ denotes a one-to-one map of $\left[1,2, \cdots, n^{d}\right]$ onto the lattice $\mathscr{L}_{d}(n)=[1,2, \cdots, n]^{d}$.

Now, it is not difficult to reinforce our belief in $(2.16)$ by deriving from it inequalities we can prove by other methods.

Indeed, as we shall soon see, by setting $\Phi(u)=|u|^{p}(p>0)$ in (2.16) we can very quickly derive from it all the classical Sobolev inequalities which state 
the integrability or smoothness of a function in terms of integrability conditions on its gradient.

Bearing in mind all these facts, $(2.13)$ as well as its $d$-dimensional analogue (2.17) now appear very plausible.

Now, it turns out that when $n$ is a power of 3 we can prove (2.17) with $c_{d}=3^{d}(d+3)^{d / 2}$.

The proof has an element of surprise in it and it stems from the insistence of my student $\mathrm{S}$. Milne in trying to find a special mapping $\varepsilon:\left[1,2, \cdots, n^{2}\right] \rightarrow \mathscr{L}_{2}(n)$ for which (2.8) may hold true for all $\pi$.

From the considerations above we see that if such a map $\varepsilon$ exists then the set $\mathscr{D}_{\mathrm{M}}(\varepsilon)$ must contain $\mathscr{R}_{\mathrm{M}^{2} / 2}$. This means that every couple $a=(i, j)$, such that $|i-j| \leqq M^{2} / 2$, must belong to $\mathscr{D}_{M}(\varepsilon)$ or, which is the same, we must have that

$$
|i-j| \leqq M^{2} / 2 \Rightarrow \overline{\varepsilon(i) \varepsilon(j)} \leqq M .
$$

Now it turns out that maps $\varepsilon:\left[1,2, \cdots, n^{2}\right] \leftrightarrow \mathscr{L}_{2}(n)$ satisfying a condition similar to (2.18) are not difficult to find. In fact, all we need to do is produce a "parametrization of $\mathscr{L}_{2}(n)$ " or better "a curve describing $\mathscr{L}_{2}(n)$ " which is Lipschitzian of order $\frac{1}{2}$. That is, a one-to-one map $\varepsilon$ of $\left[1,2, \cdots, n^{2}\right]$ onto $\mathscr{L}_{2}(n)$ which is such that

$$
\overline{\varepsilon(i) \varepsilon(j)} \leqq c|i-j|^{1 / 2} .
$$

Assuming that $\Xi$ is such a curve, the considerations above suggest that the

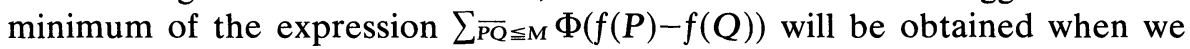
rearrange $f$ to be increasing (or decreasing) along $\Xi$.

It turns out that this is "almost" true. Indeed, using this idea we can put together a very simple proof of (2.13). And, at any rate, as we have seen, the latter is all we need to derive our desired inequalities (2.14) and (2.15). We can state this result in the following manner:

THEOREM 2.2. Let $\varepsilon$ be a one-to-one map of $\left[1,2, \cdots, n^{2}\right]$ onto $\mathscr{L}_{2}(n)$ such that

$$
\overline{\varepsilon(i) \varepsilon(j)} \leqq c|i-j|^{1 / 2} \quad \forall 1 \leqq i, j \leqq n^{2}
$$

then we have

$$
\mathscr{R}_{\mathrm{M}^{2} / \mathrm{c}^{2}}<\mathscr{D}_{\mathrm{M}}(\pi)
$$

for all $\pi$ and for all $1 \leqq M \leqq \sqrt{2} n$.

Proof. Given a map $\pi:\left[1,2, \cdots, n^{2}\right] \leftrightarrow \mathscr{L}_{2}(n)$ set $\sigma=\varepsilon^{-1} \pi$.

From the one-dimensional result, as expressed by (2.7), we get

$$
\mathscr{R}_{\mathrm{M}^{2} / \mathrm{c}^{2}}<\mathscr{D}_{\mathrm{M}^{2} / \mathrm{c}^{2}}(\sigma) \text {. }
$$

Now, from the definition,

$$
\mathscr{D}_{\mathrm{M}^{2} / c^{2}}(\sigma)=\left\{a=\left(i^{\prime}, j^{\prime}\right):\left|\varepsilon^{-1}\left(\pi\left(i^{\prime}\right)\right)-\varepsilon^{-1}\left(\pi\left(j^{\prime}\right)\right)\right| \leqq M^{2} / c^{2}\right\} .
$$


However, setting $i=\varepsilon^{-1}\left(\pi\left(i^{\prime}\right)\right)$ and $j=\varepsilon^{-1}\left(\pi\left(j^{\prime}\right)\right)$ in $(2.20)$, we get that

$$
|i-j|=\left|\varepsilon^{-1}\left(\pi\left(i^{\prime}\right)\right)-\varepsilon^{-1}\left(\pi\left(j^{\prime}\right)\right)\right| \leqq M^{2} / c^{2} \Rightarrow \overline{\pi\left(i^{\prime}\right) \pi\left(j^{\prime}\right)} \leqq M .
$$

That is we must have

$$
\mathscr{D}_{M^{2} / c^{2}}(\sigma) \subset \mathscr{D}_{M}(\pi) \text {. }
$$

Combining (2.22) and (2.23) we immediately obtain (2.21) as desired.

This result combined with Lemma 2.1 yields inequality (2.14) with $c$ replaced by $c^{2}$.

To derive (2.14), and hence (2.15), we need then exhibit for each $n$ (or at any rate for a sequence of $n$ 's) a map $\varepsilon:\left[1, \cdots, n^{2}\right) \leftrightarrow \mathscr{L}_{2}(n)$ satisfying inequalities (2.20) for some fixed constant $c$.

Now, in [4] we show that this can be done in many ways. However, it is good to point out here that if we put together these maps in a systematic manner, then they can be made to converge as $n \rightarrow \infty$ to a Peano type curve $\Xi(t):[0,1] \rightarrow[0,1] \times[0,1]$ which has the following two remarkable properties:

(a) $\Xi$ is a measure preserving map of $[0,1]$ onto $[0,1] \times[0,1]$,

(b) $\Xi$ is Lipschitzian of order $\frac{1}{2}$. That is $\overline{\Xi\left(t_{1}\right) \Xi\left(t_{2}\right)} \leqq c\left|t_{1}-t_{2}\right|^{1 / 2}$.

By a "systematic manner" we mean that we start with a fixed model for $n=n_{0}$ and then produce the corresponding model for $n=n_{0}^{k}(k=1,2, \cdots)$ in a "multiplicative" fashion.

For instance, if we start for $n_{0}=3$ with the model

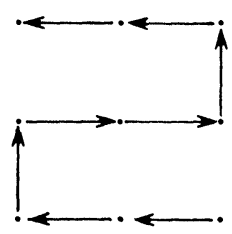

then the corresponding model for $n=3^{2}$ is obtained by ordering first the nine $3 \times 3$ subblocks according to $(2.25)$, that is:

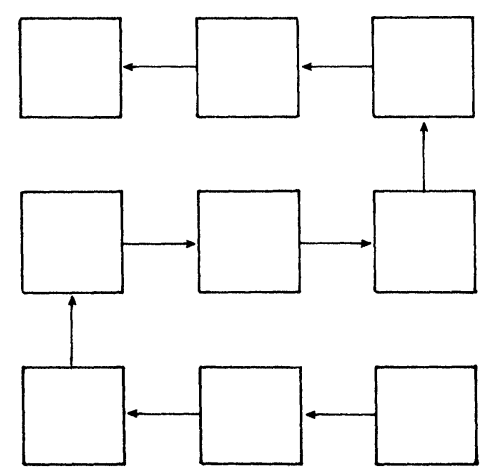


then ordering the points within the blocks according to (2.25) in a manner which is economical with distances, that is:

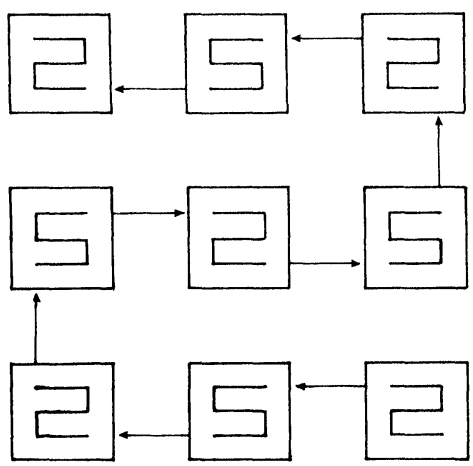

For each successive power of 3 we proceed in an analogous fashion. This is indeed Peano's original scheme for the construction of his famed curves.

In [4] we show that this scheme leads to a map $\varepsilon:[0,1] \rightarrow[0,1] \times[0,1]$ which satisfies not only $(2.24)(a)$ but also $(2.24)(b)$ with $c=3 \sqrt{5}$. This fact seems to have passed unnoticed in the literature so far.

To sum things up, we see that, roughly speaking, the combinatorial facts underlining inequality $(2.15)$ can be broken up into the combinatorial inequality in (2.6) and the existence of Peano curves satisfying (2.24).

3. Applications: Sobolev type inequalities and convergence of Fourier series. Let $f(X)$ be a measurable function on the $d$-dimensional cube $I_{d}=[0,1] \times \cdots \times[0,1](d$ times $)$. Let $f^{*}(s)$ denote the one-dimensional nonincreasing rearrangement of $f(X)$, that is, the unique. right continuous nonincreasing function on $[0,1]$ such that

$$
m\left\{s \in[0,1]: f^{*}(s) \geqq \lambda\right\}=m\left\{X \in I_{d}: f(X) \geqq \lambda\right\} \quad \forall \lambda .
$$

The end product of the combinatorial considerations of the previous section is the following

THEOREM 3.1. If $\Phi(u)=\Phi(-u) \uparrow$ as $|u| \uparrow$ then

$$
\iint_{|s-t| \leqq \delta^{d} / c_{d}} \Phi\left(f^{*}(s)-f^{*}(t)\right) d s d t \leqq \iint_{\overline{X Y} \leqq \delta} \Phi(f(X)-f(Y)) d X d Y
$$

holds for all $0 \leqq \delta \leqq \sqrt{ } d$ where $c_{d}=3^{d}(d+3)^{d / 2}$.

A complete proof of this result for every dimension can be found in [4]. The inequality in (3.1) has a number of very useful consequences. Indeed it applies in a wider class of situations than the Pólya and Szegö symmetrization principle and it is much more elementary to prove in a rigorous manner.

To give an idea of this, suppose that $f(X)$ is defined in $R_{d}$ and is periodic of period one in each of its arguments. Let $\omega_{p}(\delta, f)$ denote the $L_{p}$ modulus of 
continuity of $f$. That is

$$
\omega_{p}(\delta, f)=\left[\int_{|\rho| \leqq 1} \int_{I_{d}}|f(X+\rho \delta)-f(X)|^{p} d X d \rho\right]^{1 / p} .
$$

Let $Q_{p}\left(\Delta, f^{*}\right)$ denote the $L_{p}$ modulus of continuity of $f^{*}$; that is

$$
Q_{p}\left(\Delta, f^{*}\right)=\left[\frac{1}{\Delta} \iint_{|s-t| \leqq \Delta}\left|f^{*}(s)-f^{*}(t)\right|^{p} d s d t\right]^{1 / p}
$$

Then (3.1) for $\Phi(u)=|u|^{p}$ yields

$$
Q_{p}\left(\delta^{d} / c_{d}, f^{*}\right) \leqq c_{d, p} \omega_{p}(\delta, f)
$$

where $c_{d, p}$ is a constant depending only on $d$ and $p$.

In [2] we show that for any nonincreasing function $f^{*}(x)$ on $[0,1]$ we have

$$
\left.\begin{array}{c}
f^{*}(x)-f^{*}\left(\frac{1}{2}\right) \\
f^{*}\left(\frac{1}{2}\right)-f^{*}(1-x)
\end{array}\right\} \leqq \frac{8}{\log \frac{3}{2}} \int_{x}^{1} Q_{p}\left(\delta, f^{*}\right) \frac{d \delta}{\delta^{1+1 / p}} \quad \forall 0<x \leqq \frac{1}{2} .
$$

Combining (3.2) and (3.3) we derive the following remarkable inequality

$$
\left.\begin{array}{c}
f^{*}(x)-f^{*}\left(\frac{1}{2}\right) \\
f^{*}\left(\frac{1}{2}\right)-f^{*}(1-x)
\end{array}\right\} \leqq c_{d, p} \int_{c_{d} x^{1 / d}}^{\sqrt{d}} \omega_{p}(\delta, f) \frac{d \delta}{\delta^{1+d / p}} 0<x \leqq \frac{1}{2}
$$

This is useful insofar as it delivers us an estimate of the growth of $f^{*}$ as $x \rightarrow 0^{+}$or $x \rightarrow 1^{-}$in terms of the behavior of $\omega_{p}(\delta, f)$ for small $\delta$. For instance, if we know that

$$
\omega_{p}(\delta, f)=O(\delta)
$$

then (3.4) gives that for $p<d, f^{*}$ is in $L_{q} \forall q<d p /(d-p)$. Of course, since $f^{*} \in L_{q} \Leftrightarrow f \in L_{q}$ and $\operatorname{grad} f \in L_{p} \Rightarrow(3.5)$, we see that (3.4) extends one of the classical results of Sobolev. (See [10].)

But we are not through yet. Indeed, when the integral on the right-hand side of (3.4) is convergent as $x \rightarrow 0$, a change of scale argument (see [5]) yields that $f(X)$ is essentially continuous and $\forall X$ and $Y$ in the Lebesgue set of $f$ we have

$$
|f(X)-f(Y)| \leqq c_{d, p} \int_{0}^{\overline{X Y}} \omega_{p}(\delta, f) \frac{d \delta}{\delta^{1+d / p}} .
$$

This implies that when $\omega_{p}(\delta, f)=O(\delta)$ and $p>d$, then $f(X)$ is essentially Hölder continuous with exponent $=(p-d) / p$, and this extends another one of the Sobolev results.

The inequality in (3.6) can also be used to derive results about uniform convergence of Fourier series which seem to have passed unnoticed in the literature.

We state one of these in the one-dimensional case.

THEOREM 3.2. Let $f(x)$ be periodic of period $2 \pi$ and such that

$$
\int_{0}^{\pi} \omega_{p}(\delta, f) \frac{d \delta}{\delta^{1+1 / p}}<\infty
$$

then the partial sums $S_{n}(x, f)$ of the Fourier series of $f$ converge uniformly. 
We can give a sketch of the argument and refer to [2] for further details. We start by applying (3.6) to $S_{n}(x, f)$ and get

$$
\left|S_{n}(x, f)-S_{n}(y, f)\right| \leqq c_{p} \int_{0}^{|x-y|} \omega_{p}\left(\delta, S_{n}\right) \frac{d \delta}{\delta^{1+1 / p}}
$$

However, from M. Riesz' $L_{p}$ estimate for the partial sums we derive that

$$
\omega_{p}\left(\delta, S_{n}\right) \leqq c_{p} \omega_{p}(\delta, f) .
$$

Thus (3.7) and (3.8) give

$$
\left|S_{n}(x, f)-S_{n}(y, f)\right| \leqq c_{p} \int_{0}^{|x-y|} \omega_{p}(\delta, f) \frac{d \delta}{\delta^{1+1 / p}} .
$$

This gives that the sequence $\left\{S_{n}(x, f)\right\}$ is equicontinuous and therefore uniformly convergent. q.e.d.

We should point out that Theorem 3.2 for $1<p \leqq 2$ can be also derived from a Bernstein-type inequality. Indeed, if

$$
f \sim \sum c_{n} e^{i n x}
$$

then it can be shown (see [2]) that we also have

$$
\sum_{\nu \neq 0}\left|c_{\nu}\right| \leqq c_{p} \int_{0}^{\pi} \omega_{p}(\delta, f) \frac{d \delta}{\delta^{1+1 / p}} \quad \forall 1<p \leqq 2 .
$$

Thus the uniform convergence of $\left\{S_{n}(x, f)\right\}$ in this case follows for much better reasons. However (3.9) can be shown to be false for $p>2$ and the best we can obtain from our methods for $p>2$ is

$$
\left\|f-c_{0}\right\|_{\infty} \leqq c_{p} \int_{0}^{\pi} \omega_{p}(\delta, f) \frac{d \delta}{\delta^{1+1 / p}} .
$$

Similar results for higher dimensions and applications to path continuity of $L_{p}$ processes can be found in [11].

Before closing we would like to point out that there are also some interesting applications of (3.1) when we take $\Phi(u)=\exp u^{2}$. In fact, let $f$ be measurable in $[0,1]$ and set for each $\Phi$,

$$
\omega_{\Phi}(\delta, f)=\inf \left\{\lambda: \frac{1}{\delta} \iint_{|x-y| \leqq \delta} \Phi\left(\frac{f(x)-f(y)}{\lambda}\right) d x d y \leqq \Phi(1)\right\} .
$$

This might be thought of as the $\Phi$-modulus of continuity of $f$. P. DeLand [3] showed that there are inequalities analogous to (3.3) and (3.6) for each convex $\Phi$.

For the case $\Phi(u)=\exp u^{2}$ the following result holds:

THEOREM 3.3. Let $f$ be measurable in $[0,1]$ and such that

$$
\int_{0}^{1} \omega_{\exp u^{2}}(\delta, f) \frac{d \delta}{\delta \sqrt{\log \delta^{-1}}}<\infty
$$


then $f$ is essentially continuous and for a universal constant $c$,

$$
|f(x)-f(y)| \leqq c \int_{0}^{|x-y|} \omega_{\exp u^{2}}(\delta, f) \frac{d \delta}{\delta \sqrt{\log \delta^{-1}}}
$$

holds at all points of the Lebesgue set of $f$.

One of the consequences of this result is the following extension of a classical theorem of Paley-Zygmund-Salem on uniform convergence of random Fourier series.

THEOREM 3.4. Let $\left\{c_{n}\right\}$ be a sequence of constants such that

$$
\int_{0}^{\pi}\left(\sum\left|c_{n}\right|^{2} \sin ^{2} n \delta\right)^{1 / 2} \frac{d \delta}{\delta \sqrt{\log \delta^{-1}}}<\infty .
$$

Then the random Fourier series $\sum \pm c_{n} e^{i n x}$ converges uniformly with probability one.

We should point out that this result was also obtained by Marcus and Jain [9] by entirely different methods.

\section{REFERENCES}

1. A. M. Garsia, On the smoothness of functions satisfying certain integral inequalities, Functional Analysis (Proc. Sympos., Monterey, Calif., 1969), Academic Press, New York, 1970, pp. 127-162. MR 42 \#8267.

2. - A remarkable inequality and the uniform convergence of Fourier series, Indiana Univ. Math. J. (to appear).

3. A. M. Garsia and P. DeLand, Moduli of continuity for exponential Lipschitz classes (to appear).

4. A. M. Garsia and S. Milne, Peano curves and smoothness of functions (to appear).

5. A. M. Garsia and E. Rodemich, Monotonicity of certain functionals under rearrangements, Ann. Inst. Fourier (Grenoble) 24 (1974), fasc. 2, 116.

6. A. M. Garsia, E. Rodemich and H. Rumsey, Jr., A real variable lemma and the continuity of paths of some Gaussian processes, Indiana Univ. Math. J. 20 (1970/71), 565-578. MR 42 \#2534.

7. R. K. Getoor and H. Kesten, Continuity of local times for Markov processes, Compositio Math. 24 (1972), 277-303. MR 46 \#10075.

8. C. A. Greenhall, Growth and continuity of functions satisfying quadratic integral inequalities, Indiana Univ. Math. J. 21 (1971/72), 157-175. MR 44 \# 5420.

9. M. B. Marcus and N. C. Jain, Sufficient conditions for the continuity of stationary Gaussian processes and applications to random series of functions, Ann. Inst. Fourier (Grenoble) 24 (1974), fasc. 2, 117-141.

10. L. Nirenberg, On elliptic partial differential equations, Ann. Scuola Norm. Sup. Pisa (3) 13 (1959), 115-162. MR 22 \#823.

11. T. W. Park, Sobolov type inequalities and path continuity of $L_{p}$ processes with multidimensional time parameter (to appear).

12. C. Preston, Continuity properties of some Gaussian processes, Ann. Math. Statist. 43 (1972), 285-292. MR 46 \#6436.

13. H. Taylor, Rearrangements of incidence tables, J. Combinatorial Theory Ser. A 14 (1973), 30-36. MR 47 \#8322.

P.O. Box 295, Rancho Sante Fe, California 92067 